\title{
STERNOCLAVICULAR GRAFT VERSUS COSTOCHONDRAL GRAFT FOR RECONSTRUCTION ANKYLOSED TEMPOROMANDIBULAR JOINT IN A CHILD (CLINICAL AND RADIOGRAPHIC COMPARATIVE STUDY)
}

\author{
Abdelbadia Abdallah Abdelmabood* and Abdelaziz Baiomy Abdullah**
}

\begin{abstract}
Aim: To evaluate the feasibility of using sternoclavicular graft (SCG) versus costochondral graft (CCG) for TMJ reconstruction in TMJ ankylosis in child.

Patients and methods: A total of 20 patients having unilateral bony TMJ ankylosis underwent gap arthroplasty then reconstruction of TMJ with SCG (Group I) or CCG (Group II). Pre-operative and post-operative clinical and radiographic evaluation were done to assess maximum mouth opening, range of mandibular movements, ramus height, donor and recipient sites complications for 24 month follow up at least.
\end{abstract}

Results: Statistical analysis shows that postoperative measurements of different mouth movements increased comparing preoperative measurements in both groups without any significant difference between groups. While postoperative complications occurrence were higher in Group II than group I in pleural tear, pain, overgrowth, and facial asymmetry aspects with high statistical significant difference for pleural tear complication ( $\mathrm{p} \leq 0.01$ ). In opposite side, group I showed higher occurrence for skin scare as compared to group II with statistical significant difference. Postoperative radiographic assessment presented that the most measurements of both groups were better than the preoperative values without any statistical significant difference between groups $(\mathrm{p}>0.05)$.

Conclusion : The present study concludes SCG is a versatile and simple alternative graft to the traditional CCG for TMJ reconstruction with minimal complications in children with TMJ ankylosis.

KEYWORDS: Temporomandibular joint ankylosis, gap arthroplasty, TMJ reconstruction Sternoclavicular graft, Costochondral graft, growth.

\footnotetext{
* Associate Professor, Oral Maixofacial Surgery Department, Faculty of Dental Medicine and Oral Surgery; Zagazig University.

** Associate Professor, Oral Maixofacial Surgery Department, Faculaty of Dental Medicine, Al-Azhar University, Assuit branch.
} 


\section{INTRODUCTION}

Temporomandibular joint (TMJ) ankylosis is one of the most distressing clinical conditions resulting in loss of jaw function and impairment of growth ${ }^{(1)}$. TMJ ankylosis defined as a joint problem which refers to bone or fibrous adhesion of the anatomic joint compartments, resulting in decrease or absent of TMJ movements ${ }^{(2)}$.

Several causes have been attributed to the formation of TMJ ankylosis, of which previous trauma, previous TMJ surgery, arthritis, and infection. It can be congenital, and in some cases, idiopathic. The most common etiology of TMJ ankylosis is previous trauma, with the second being infection ${ }^{(3-5)}$.

Occurrence of TMJ ankylosis in a child had several complications including issues with airway maintenance, feeding difficulties and speech development alterations. Besides, it meddles with the facial skeletal and dento-alveolar development in the growing child prompting facial asymmetry ${ }^{(5,6)}$.

Accordingly, to restore joint function, and allow for mandibular growth, the various techniques for surgical correction of TMJ ankylosis are developed such as Gap arthroplasty,Interpositional arthroplasty, and Joint reconstruction ${ }^{(7-9)}$. Numerous materials have been suggested for condylar reconstruction subsequent to eliminating the ankylotic mass, for example, costochondral, sternoclavicular, posterior border of mandibular ramus, fibular, coronoid, and metatarsophalangeal, alloplastic graft, and as of late, condylar distraction osteogenesis ${ }^{(7,10-13)}$.

Traditionally, the costochondral grafts (CCG) have been used for reconstruction of TMJ in ankylosis patients, because of it is naturally viable like any autogenous graft, effectively functional, especially when contouring the cartilaginous part to fit into the glenoid fossa. Additionally, it has the capability for remolding into an adaptive mandibular condyle, and there is consistently a potential at the donor site to grow and regenerate. However, it has several drawbacks such as the unpredictable growth, and fracture of grafts. This enhanced researchers to search for another alternative ${ }^{(10,11,14)}$.

Sternoclavicular graft (SCG) and TMJ are comparable anatomically and physiologically. The Sternoclavicular joint articulation has a growth center and an interarticular fibrocartilage articular disc that mimics the meniscus of the TMJ. When an entire sternoclavicular joint is utilized as bone graft, the two neighboring synovial compartments and the strong fibrous capsule look like those in the $\mathrm{TMJ}^{(14,15)}$.

Several studies ${ }^{(14,16)}$ attempted to compare SCG with CCG in reconstruction of temporomandibular joint. But there was controversy about superiority of one on another graft. This may be because of some drawbacks of these studies such as limited sample size and short follow up period. Therefore, the current study is an attempt with longer follow up and larger sample size to evaluate efficacy of sternoclavicular graft versus costochondral graft in reconstruction of ankylosed TMJ of children.

\section{PATIENTS AND METHODS}

Twenty children with average 11 years of age were included in our study. They were diagnosed of unilateral bony TMJ ankylosis at the outpatient clinic at Oral and Maxillofacial Surgery department, faculty of dental medicine, Al-Azhar University, (Assiut, Boys).

The clinical study extended from March 2018 till March 2020 year. Study procedures were explained to parents of children and they signed an informed consent form before the study. The study was conducted according to rules of ethics declared by Helsinki, and ethical committee approval was obtained from ethics committee of dental medicine faculty, Al -Azhar university, Assuit branch (code: AUAREC2020118-7). 


\section{Selection criteria}

-- Inclusion criteria: All selected patients had unilateral TMJ bony ankylosis, no previous treatment for ankylosis, no severe facial asymmetry, and their age below 14 years

-- Exclusion criteria: Patients with bilateral TMJ ankylosis, patient had no local or systemic contraindication for operation, and Patient did not complete the follow up protocol of at least 24 months

\section{Patient grouping}

Patients classified randomly into the following two equal groups using online software (https:// www. randomizer.org):

Group I-10 patients underwent gap arthroplasty and reconstruction with SCG

Group II- 10 patients underwent gap arthroplasty and reconstruction with CCG

\section{Preoperative assessment}

Pre-operative evaluation incorporated a thorough history and physical examination to decide the reason of ankylosis, measurement of maximal incisor opening (MIO), occlusion, and facial asymmetry (fig1a\&2a). Preoperative radiographic analysis included panoramic, cephalograms, CT scan (fig $1 \mathrm{~b} \& 2 \mathrm{~b}$ ), and posteroanterior (PA) view of chest.

\section{Operative Procedure}

All surgical procedures were done by the same operator under general anaesthesia following strict asepsis. After induction of anaesthesia, application of antiseptic, and standard surgical draping the TMJ was approached through an endaural incision (fig. 1c \& 2c). The superficial temporal fascia is distinguished, and its plane followed inferiorly and anteriorly to arrive at the zygomatic arch and capsule of the TMJ. Arthrectomy was performed making a gap of $1.5 \mathrm{~cm}$. The transoral temporalis tendon and pterygomasseteric sling were released, the masseter reflected, and coronoidectomy done if the interincisal distance was $<35 \mathrm{~mm}$.

Immediate reconstruction was done with graft around $5 \mathrm{~mm}$ shorter than the length of resected segment to keep up the gap between the superior part of the graft (condylar head) and the glenoid fossa.

In Group I, SCG was harvested from the ipsilateral side by utilizing supraclavicular incision, 1-2 cm above the clavicle ( fig. 1d\&e), and in Group II, CCG was harvested from the contralateral side by making submammary incision at the 5 th or 6th rib, starting around $4 \mathrm{~cm}$ from the midline ( fig. $2 \mathrm{~d} \& \mathrm{e})$.

The grafts were fixed on the lateral aspect of the ramus by at least two titanium screws with 10$\mathrm{mm}$ length and $1.5 \mathrm{~mm}$ dimeter in both the groups. Temporalis fascia was inserted in the gap and fixed with 3-0 vicryl suture to prevent reankylosis. Wound closure was done in layer by layer manner where the muscle layer was closed, and a suction drain was placed and secured with suture. Skin was sutured by giving interrupted sutures using 3-0 black silk. Pressure dressing was placed and changed each 12 $\mathrm{h}$ for 4-5 days postoperatively.

All patients were placed in inter-maxillary fixation (IMF) for one week for stabilization of the graft. Aggressive mouth opening physiotherapy was begun after the $7^{\text {th }}$ post-operative day and proceeded till the patients learnt the exercise and continued it by themselves. Every patient had a custom-made acrylic bite block that was fitted to the occlusal surface of the molar-premolar segment after ideal mouth opening had been accomplished and was subsequently removed from the mouth after all reflexes have been regained. Each acrylic bite block was utilized for 6 months to maintain and improve the intraoperative mouth opening. 


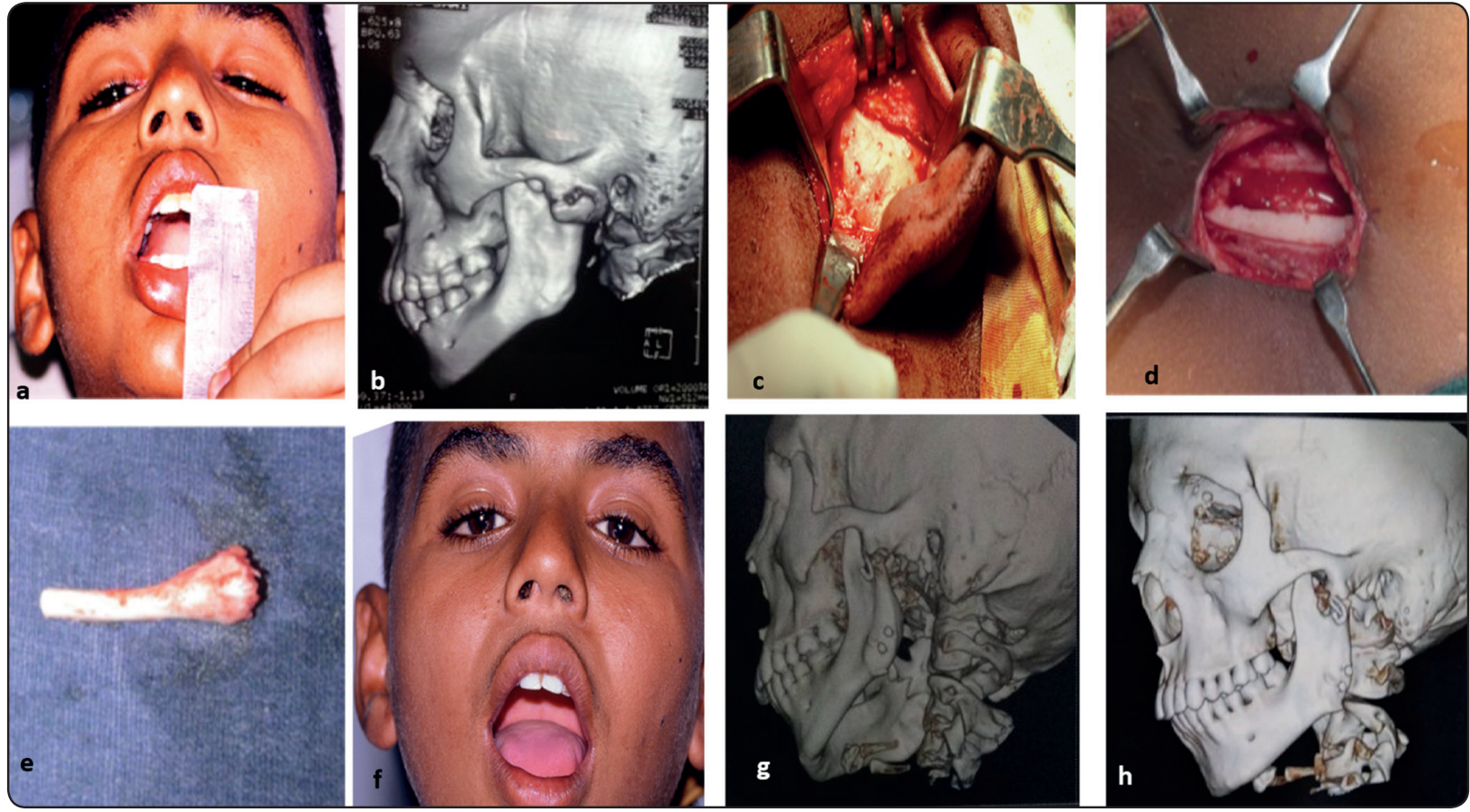

Fig. (1): (a) Preoperative mouth opening measurment (b) 3D CT showed bony ankylosis in group I (c) Endaural surgical approach showing ankyltic mass(d) Harvesting of sternoclavicular graft. (e) Harvested SCG (f) Postoperative maximal mouth opning (g) 3D CT showing fixed harvested SCG at postopertive 6 months (g) 3D CT showing SCG at postopertive 24 months .
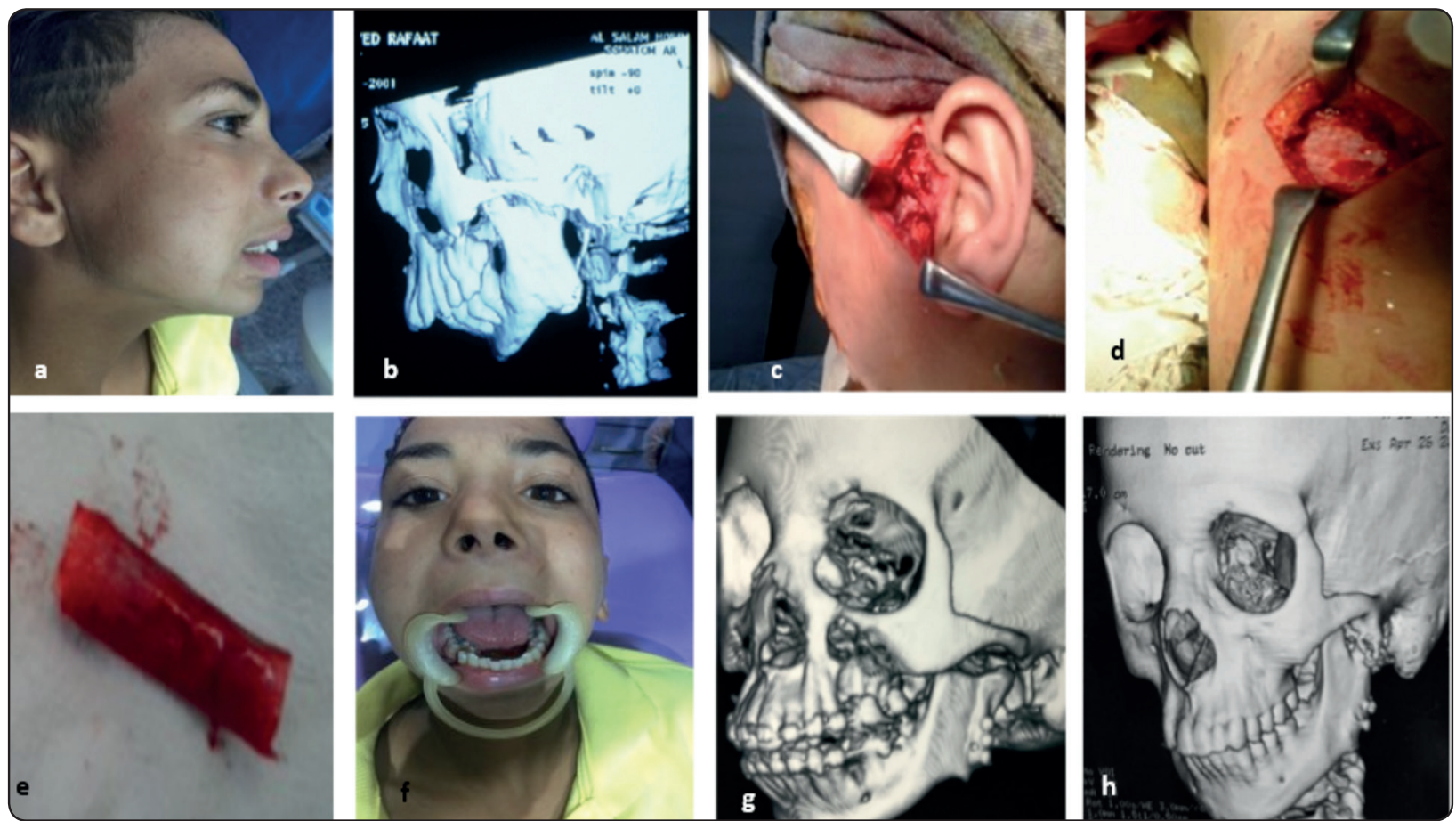

Fig. (2) (a) Preoperative limited mouth opening (b) 3D CT showed bony ankylosis in group II (c) Endaural surgical approach (d) Submammary incision. (e) Harvested CCG (f) Postoperative maximal mouth opning (g) 3D CT showing fixed harvested CCG at postopertive 6 months (h) 3D CT showing CCG at postopertive 24 months 


\section{Post-Operative Assessment}

Clinical boundaries surveyed were: mouth opening, lateralexcursion, and protrusive movements after $1^{\text {st }}$ week, $1^{\text {st }}, 3 \mathrm{rd}, 6^{\text {th }}, 12^{\text {th }}$, and 24 months follow-up (fig.1f \&2f). Also, the complications of graft harvest site in both the groups were assessed. Postoperative radiographs were taken to evaluate the functional adaptation of the graft at surgical side. Patients were checked on regular follow-ups for 6,12, 24 months (fig.1g,h \& fig.2g,h). PA chest radiograph was done to survey the thickness of the clavicle or rib, and any abnormality if present.

\section{Statistical analysis}

The study variables data were gathered and coded utilizing the statistical package of social sciences (SPSS, version 24, Chicago, Illinois, USA) program for statistical analysis. Descriptive and inferential analyses were performed using t-test and chi-square test for association. P-value was set at or below 0.05 to define significance.

\section{RESULTS}

Twenty three patients were recruited in the study (only 20 patients have been shown on the assigned follow up time and included in the results). Twenty patients suffered from unilateral bony TMJ ankylosis were included in the present study and their age ranged between 9 and 13 years (average 11 years). Males predominated with a percentage of $55 \%$ of the cases. Seven TMJ ankylosis were at right side and 13 at left side. The mean age was $10.5 \pm 2.38$ in group I and 11.5 \pm 2.54 in group II with no significant differences between the two groups. Trauma was the main etiological factor in 15 patients (75\% of all cases), while middle ear infection was the etiological factor in 5 patients (25\% of all cases) (Table 1) .

\section{Clinical observations}

Postoperative complications are summarized in (Table 2). Regarding donor site complications, there was a plural tear noticed during operation only in 3 patients (30\%) of the group I which was repaired immediately without pneumothorax. Clavicular fracture occurred in only 1 cases $(10 \%)$ of the group I. Pain at donor site after $1^{\text {st }}$ postoperative month was evident in group II ( $40 \%$ of cases) than group I (10\%) with statistical significant difference $(\mathrm{p} \leq 0.05)$.In the opposite side skin scare at donor site was more noticeable in group I (40\%) than group II ( $20 \%$ ) with statistical significant difference $(\mathrm{p} \leq 0.05)$. In the present study, all the cases showed complete regeneration of the donor site during the follow-up.

TABLE (1): Demographic data of the patients

\begin{tabular}{|c|c|c|c|c|}
\hline \multicolumn{2}{|c|}{ Variables } & SCG & CCG & $p$ value \\
\hline \multirow{2}{*}{ Sex (no. of cases) } & Male & 5 & 6 & \multirow{2}{*}{0.81} \\
\hline & Female & 5 & 4 & \\
\hline \multicolumn{2}{|l|}{ Range of age (years) } & $9-12($ mean $=10.5 \pm 2.38)$ & $10-13($ mean $=11.5 \pm 2.54)$ & 0.72 \\
\hline \multirow{3}{*}{$\begin{array}{l}\text { Cause of ankylosis } \\
\text { (no. of cases) }\end{array}$} & Trauma & 8 & 7 & \multirow{3}{*}{0.95} \\
\hline & Infection & 2 & 3 & \\
\hline & Others & 0 & 0 & \\
\hline \multirow{2}{*}{ Side (no. of cases) } & RT & 5 & 2 & \multirow{2}{*}{0.75} \\
\hline & $\mathrm{LF}$ & 5 & 8 & \\
\hline
\end{tabular}


Regards to recipient-site complications, there was no signs of graft rejection, wound infection, or esthetic problems encountered in any case. There was no permanent facial nerve damage, except in some cases the frontal and temporal branches of the facial nerve were temporarily affected in 2 cases in group I and 3 cases in group II, which resolved in period ranged from 3 to 6 months after uptake medications and physiotherapy sessions. At end study all cases showed no bone graft fracture or reankylosis except one case in group II for each item . In the present study, over growth complication was more observed in group II (40\%) than group I (10\%) with statistical significant difference $(\mathrm{p} \leq 0.05)$ between both groups. Regarding joint sounds, joint clicking observed in one patient of group I (10\%) and one patient of group II (10\%) with statistical insignificant difference $(\mathrm{p} \geq 0.05)$.

In, current study, postoperative complain of mandibular deviation during opening was prominent in group II $(50 \%)$ with statistical significant difference $(\mathrm{p} \leq 0.05)$ than group I $(20 \%)$. Which was improved later in some case with the help of intermaxillary elastics. Also, facial asymmetry was noticeable in 5 cases of group II $(50 \%)$ versus 2 cases of group I (20\%) with statistical significant difference $(\mathrm{p} \leq 0.05)$ between groups.

Regarding TMJ movements, group II had higher values than group I in postoperative maximum mouth opening, lateral excursion toward affected side and protrusive movement without any significant differences $(p>0.05)$ between groups (fig. $1 \mathrm{~g}$ \& 2. g). While group I had higher values than group I in postoperative lateral excursion toward non affected side but without any significant differences ( $p>0.05)$ between groups. Where the most measurements of both groups were significantly better than the preoperative values. Satisfactory mouth opening, lateral excursion and protrusive movements were achieved in all the patients at 6 month, they increased progressively in most patients with active exercises along follow up (Table 3 ).

TABLE (2) : Postoperative complications in groups

\begin{tabular}{|c|c|c|c|c|}
\hline \multicolumn{2}{|c|}{ Variables } & \multirow{2}{*}{$\begin{array}{c}\text { SCG } \\
\frac{(\text { no. of cases })}{0}\end{array}$} & \multirow{2}{*}{$\begin{array}{c}\begin{array}{c}\mathbf{C G} \\
(\text { no. of cases) }\end{array} \\
3\end{array}$} & \multirow{2}{*}{$\begin{array}{c}\boldsymbol{p} \text { value } \\
0.001^{* *}\end{array}$} \\
\hline Donor site complications & Pleural tear & & & \\
\hline & clavicular fracture & 1 & 0 & 0.561 \\
\hline & Pain at $1^{\text {st }}$ month & 1 & 4 & $0.042 *$ \\
\hline & Skin scar & 4 & 2 & $0.057 *$ \\
\hline \multirow[t]{4}{*}{ Recipient-site complications: } & Facial nerve palsy & 2 & 3 & 1.671 \\
\hline & Bone graft fracture & 0 & 1 & 0.913 \\
\hline & Re-ankylosis & 0 & 1 & 0.071 \\
\hline & Over growth & 1 & 4 & $0.035 *$ \\
\hline \multicolumn{2}{|l|}{ Joint sounds } & 0 & 0 & 2.917 \\
\hline \multicolumn{2}{|c|}{ Postoperative mandibular deviation on opening (mm) } & 2 & 5 & $0.045^{*}$ \\
\hline \multicolumn{2}{|l|}{ Facial asymmetry } & 2 & 5 & $0.051 *$ \\
\hline
\end{tabular}




\section{Radiographic observations}

Table 4 presented a comparison between the mean height of ramus of mandible of groups, statistically non- significant difference was found. Where the most measurements of both groups were better than the preoperative values with statistical significant difference in group I $(\mathrm{p} \leq 0.05)$. Regards to adaptation of graft to condyle and glenoid fossa, our results showed high percent of patients with proper adaptation of graft to condyle and glenoid fossa without any statistical significant difference between both group $(\mathrm{p}>0.05)$

TABLE (3): Assessment of different mouth movements in groups at different intervals

\begin{tabular}{|c|c|c|c|c|}
\hline Variables & Periods & SCG & CCG & $P$ value \\
\hline \multirow[t]{4}{*}{ Maximum mouth opening (mm) } & Preoperative & $7.42 \pm 1.70$ & $8.27 \pm 2.28$ & 1.420 \\
\hline & 6 month & $32.30 \pm 1.98$ & $35.80 \pm 0.73$ & 0.435 \\
\hline & 12 month & $33.209 \pm 1.08$ & $37.40 \pm 1.04$ & 0.142 \\
\hline & 24 month & $35.53 \pm 0.92$ & $39.13 \pm 0.96$ & 0.761 \\
\hline \multirow[t]{4}{*}{ Lateral excursion at the affected side (mm) } & Preoperative & $0.50 \pm 0.80$ & $0.60 \pm 0.58$ & 1.200 \\
\hline & 6 month & $2.67 \pm 0.62$ & $3.24 \pm 0.51$ & 1.000 \\
\hline & 12 month & $3.79 \pm 0.62$ & $4.42 \pm 0.25$ & 1.000 \\
\hline & 24 month & $4.13 \pm 0.65$ & $5.01 \pm 0.45$ & 0.854 \\
\hline \multirow{4}{*}{$\begin{array}{l}\text { Lateral excursion at the non-affected side } \\
(\mathrm{mm})\end{array}$} & Preoperative & $0.51 \pm 0.40$ & $0.58 \pm 0.24$ & 0.960 \\
\hline & 6 month & $2.50 \pm 0.60$ & $1.20 \pm 0.58$ & 0.954 \\
\hline & 12 month & $2.70 \pm 0.88$ & $1.40 \pm 0.68$ & 0.951 \\
\hline & 24 month & $2.65 \pm 0.71$ & $1.83 \pm 0.50$ & 0.967 \\
\hline \multirow[t]{4}{*}{ Protrusive movement (mm) } & Preoperative & $0.50 \pm 0.34$ & $0.20 \pm 0.20$ & 1.000 \\
\hline & 6 month & $0.72 \pm 0.42$ & $0.82 \pm 0.25$ & 1.000 \\
\hline & 12 month & $0.79 \pm 0.50$ & $1.45 \pm 0.46$ & 1.067 \\
\hline & 24 month & $1.45 \pm 0.39$ & $1.86 \pm 0.59$ & 1.108 \\
\hline
\end{tabular}

$* *$ High statistically significance when $p \leq 0.01$

* Statistically significance when $p \leq 0.05$

TABLE (4) : Comparison between the mean height of ramus of mandible and adaptation of graft to condyle and glenoid fossa of both groups

\begin{tabular}{|c|c|c|c|c|}
\hline \multicolumn{2}{|c|}{ Variables } & SCG & CCG & $p$ value \\
\hline \multirow[t]{4}{*}{ Height of ramus (mm) } & Preoperative & $39.00 \pm 1.25$ & $42.00 \pm 1.27$ & 0.424 \\
\hline & Postoperative $24 \mathrm{~m}$ & $45.00 \pm 1.66$ & $44.24 \pm 1.22$ & 0.784 \\
\hline & Mean difference & 8 & 2 & ---------- \\
\hline & $P$ value & $0.032 *$ & 0.710 & ----------- \\
\hline \multicolumn{2}{|c|}{ Adaptation of graft to condyle and glenoid fossa (no. of cases) } & 7 & 8 & 0.065 \\
\hline
\end{tabular}




\section{DISCUSSION}

TMJ reconstruction after ankylosis management remains one of the most challenges of maxillofacial surgeons, with a variety of autogenous and alloplastic techniques available. Several autogenous grafts are available for condylar reconstruction after the ankylotic mass releasing such as costochondral, sternoclavicular, fibular, coronoid, and metatarsophalangeal ${ }^{(7,10-13)}$.

Authors ${ }^{(7,14,15)}$ preferred CCG for reconstruction of condyle in growing patients, where growth capacity is compatible with the ascending branch of the mandible. Their bony part is used to reconstruct the condylar neck and ramus while the cartilaginous portion occupy newly constructed glenoid fossa. Accordingly, the bone cartilage junction provides a center with growth potential. However, CCG had several drawbacks such as unpredictable growth, warpage, and fracture of the CCG . Therefore, researchers developed several trials to search for an alternative $^{(17,18)}$.

SCG is morphologically and histologically is very similar to TMJ as they are the only two synovial joints covered with fibrocartilage. Moreover, there are no significant differences in the potential for mandibular growth between reconstructions with sternoclavicular and with costochondral grafts ${ }^{(14,15)}$. This similarity of the joints has encouraged the surgeons to use Sternoclavicular graft (SCG) as an alternative to CCGs.

In the other side, Seth $\mathrm{S}$ et $\mathrm{al}^{(16)}$ concluded the superiority of costochondral graft over sternoclavicular graft in terms of growth and function. This controversy was a cause to developed our study to compare between SCG and CCG in reconstruction ankylosed TMJ in children through clinical and radiographic aspects .

In the current study, age of patients ranged from 9 to 13 years in accordance with studies of Babu et $\mathrm{al}^{(19)}$, and Bello et $\mathrm{al}^{(20)}$ that observed that ankylosis of the TMJ usually develops before the age of 10 years, but can be found at any age; the incidence peaks in patients with average 10 years. Shashikiran et al ${ }^{(20)}$ concluded that TMJ ankylosis is slightly more common in boys than girls, with a ratio of 1.4:1.2. this in acceptance with our study.

Present study conducted on children with unilateral ankylosis where it has been reported to be more common than bilateral, with a ratio of 1.5:1. Left joint was more affected than the right joint, though our sample size without any significant difference, this may be explained by reflex mechanisms as majority of the population is right handed which is matched with Kavin et $\mathrm{al}^{(21)}$.

At all postoperative intervals, the most TMJ movement measurements of both groups were significantly better than the preoperative values. Although, there were not statistical insignificant difference between groups, group II had higher values than group I in postoperative maximum mouth opening, lateral excursion toward affected side and protrusive movement and group I had higher values than group I in postoperative lateral excursion toward non affected side. These results were in accordance with Divya et al ${ }^{(14)}$, and Seth et al ${ }^{(16)}$.

Although, most authors observed that growth potential is an important advantage of CCG in rebuilding aneocondyle, particularly in growing patients ${ }^{(18,23)}$ but our results showed that SCG also has the same potential for continued growth in accordance with Passi et al ${ }^{(11)}$.The explanation of these results is because of histological similarity of sternoclavicular joint to temporomandibular joint.

Regarding to over growth of graft, it was more observed in group II than group I with statistical significant difference between both groups. Accordingly, facial asymmetry was more noticeable in group II $(50 \%)$ versus group I $(20 \%)$ with statistical significant difference $(\mathrm{p}<0.05)$ between groups. This is in the same side with Balaji et al ${ }^{(17)}$ and Baek et al ${ }^{(24)}$ studies that observed mandibular overgrowth on the grafted site which leaded to push the chin toward the unaffected side causing esthetic problems and limitations in mandibular movements. 
Complications at the donor site included plural tear and pain in our study were consistent with previous reports. ${ }^{(23,25)}$ Where, SCG avoided these complications. Although, skin scare at donor site was more noticeable in SCG group than group with statistical significant difference. This in accordance with Seth et al ${ }^{(16)}$.

In current study, at end study none of the cases in either group showed infection, nerve damage, bone graft fracture or re-ankylosis except one case in group II for bone graft fracture and re-ankylosis items. Beside, all the cases using the SCG and CCG showed complete regeneration of the clavicle and rib, respectively, during the follow-up.

On comparing the mean height of ramus of mandible and adaptation of graft to condyle and glenoid fossa between both the groups, statistically no significant difference was found. Where the most measurements of both groups were better than the preoperative values with statistical significant difference in group I $(\mathrm{p}<0.05)$. This is in opposite direction to Seth et al (16) who observed the height of ramus was $10.5 \%$ higher in CCG group as compared to SCG group explained this by bonecartilage junction which provides a center with growth potential. The our explanation for this, low sample size and short time follow up of this study are drawbacks of this study. Moreover, both SCG and CCG were morphologically and histologically similar to TMJ therefore, no difference between two grafts in adaptation of graft to condyle and glenoid fossa in accordance with Passi et al (11) and Divya et al ${ }^{(14)}$.

\section{CONCLUSION}

TMJ reconstruction has been one of the greatest challenges to surgeons over decades, because of TMJ is a complex anatomic structure. Postoperative defects of TMJ can lead to poorer esthetic and functional outcomes. From the results of the present study on large number of cases and longtime evaluation, we can conclude that the
SCG as a graft material to reconstruct the condyle after gap arthroplasty in TMJ ankylosis of child produced good and comparable results to the CCGs. Accordingly, SCG may be used as a good alternative for CCG in terms of growth, adaptability and range of functional movement with little donor site morbidity and postoperative complications.

\section{FUND}

This research did not receive any specific grant from funding agencies in the public, commercial, or not-for-profit sectors.

Conflict of Interest: The authors declare that they have no conflict of interest.

\section{REFERENCES}

1. Vasconcelos BC, Bessa-Nogueira RV, Cypriano RV. Treatment of temporomandibular joint ankylosis by gap arthroplasty. Med Oral Patol Oral Cir Bucal 2006;11:66-9.

2. Erol B, Tanrikulu R, Gorgun B.A clinical study on ankylosis of the temporomandibular joint. J Craniomaxillofac Surg. 2006;34(2):100-6.

3. Zhi K, Ren W, Zhou H, Gao L, Zhao L, Hou C, Zhang Y. Management of temporomandibular joint ankylosis: 11 years' clinical experience. Oral Surg Oral Med Oral Pathol Oral Radiol Endod. 2009;108(5):687-92.

4. Elgazzar RF, Abdelhady AI, Saad KA, Elshaal MA, Hussain MM, Abdelal AE, Sadakah AA. Treatment modalities of TMJ ankylosis: experience in Delta Nile, Egypt. Int J Oral Maxillofac Surg. 2010;39(4):333-42.

5. Hegab A, El Madawy A, Shawkat WM. Congenital maxillomandibular fusion: a report of three cases. Int $\mathrm{J}$ Oral Maxillofac Surg. 2012;41(10):1248-52.

6. Yew CC, Ab Rahman S, Alam MK. Temporomandibular joint ankylosis in a child: an unusual case with delayed surgical intervention. BMC Pediatrics 2015; 15:169-77.

7. Kaban LB, Bouchard C, Troulis MJ. A protocol for management of temporomandibular joint ankylosis in children. J Oral Maxillofac Surg 2009;67:1966-78.

8. Movahed, R. and L.G. Mercuri, Management of temporomandibular joint ankylosis. Oral and Maxillofacial Surgery Clinics, 2015. 27(1): p. 27-35. 
9. Topazian RG. Comparison of gap and interposition arthroplasty in the treatment of temporomandibular joint ankylosis. J Oral Surg. 1966;24(5):405-9.

10. Singh V, Verma A, Kumar I, Bhagol A: Reconstruction of ankylosed temporomandibular joint. sternoclavicular grafting as an approach to management. Int $\mathrm{J}$ Oral Maxillofac Surg 2011;40:260-5.

11. Passi D, Singh G, Singh S, Mehta G, Dutta S, Sharma $\mathrm{S}$. Advances in temporomandibular joint reconstruction in TMJ ankylosis : Our experiences and literature review. Int J Dent Res 2014; 2, 45-9.

12. Zhu SS, Hu J, Li J, Luo E, Liang X, Feng G. Free grafting of autogenous coronoid process for condylar reconstruction in patients with temporomandibular joint ankylosis. Oral Surg Oral Med Oral Pathol Oral Radiol Endod.2008;106(5),662-7.

13. D. Mehrotra S. Kumar S. Dhasmana. . Hydroxyapatite/ collagen block with platelet rich plasma in temporomandibular joint ankylosis: a pilot study in children and adolescents. British Journal of Oral \& Maxillofacial Surgery, 2012; 50 (8), 774-778

14. Divya Mehrotra, R. Pradhan, S. Mohammad, S. Kumar (2011) Complications associated with different surgical modalities for management of temporomandibular ankylosis in a series of 791 cases, Asian Journal of Oral and Maxillofacial Surgery 2011; 23, (3), 105-160

15. Daniels S, Ellis 3rd E, Carlson DS. Histologic analysis of costochondral and sternoclavicular grafts in the TMJ of the juvenile monkey. J Oral Maxillofac Surg 1987;45,675-83.

16. Seth S, Gupta H, Kumar D, Agarwal R, Gupta S, Mehra H, Shankar NS, Singh J. Sternoclavicular Graft Versus Costochondral Graft In Reconstruction of Ankylosed Temporomandibular Joint. J. Maxillofac. Oral Surg. 2019; 18(4):559-566
17. Balaji SM, Balaji P. Overgrowth of costochondral graft in temporomandibular joint ankylosis reconstruction: A retrospective study. Indian J Dent Res 2017;28:169-74.

18. Zheng LW, MaL, ShiXJ, Zwahlen RA, Cheung LK. Comparison of distraction osteogenesis versus costochondral graft in reconstruction of temporomandibular joint condylectomy with disc preservation. J Oral Maxillofac Surg 2011;69:409-17.

19. Babu L, Jain MK, Ramesh C, Vinayaka N. Is aggressive gap arthroplasty essential in the management of temporomandibular joint ankylosis? A prospective clinical study of 15 cases. Br J Oral Maxillofac Surg 2013;51: 473-8.

20. Bello SA, Aluko Olokun B, Olaitan AA, Ajike SO. A etiology and presentation of ankylosis of the temporomandibular joint: report of 23 cases from Abuja, Nigeria. Br J Oral Maxillofac Surg 2012;50:80-4.

21. Shashikiran ND, Reddy SV, Patil R, Yavagal G. Management of temporo-mandibular joint ankylosis in growing children. J Indian Soc Pedod Prevent Dent 2005;23: 35-7.

22. Kavin T, John R, Venkataraman SS . The role of three dimensional computed tomography in the evaluation of temporomandibular joint ankylosis. J Pharm Bioallied Sci 2012.4(Suppl 2):S217-S220

23. Khadka A, Hu J. Autogenous grafts for condylar reconstruction in treatment of TMJ ankylosis:current concepts and considerations for the future. Int $\mathrm{J}$ Oral Maxillofac Surg 2012;41:94-102.

24. Baek R M, Song Y T. Overgrowth of acostochondral graft in reconstruction of the temporomandibular joint. Scand J Plast Reconstr Surg Hand Surg 2006;40:179-85.

25. Skouteris CA, Sotereanos GC. Donor site morbidity following harvesting of autogenous rib grafts. J Oral Maxillofac Surg 1989;47:808-12 